\title{
Factores que influyen en el estilo de vida de los funcionarios de una universidad estatal de Costa Rica: nivel educativo, estado civil y número de niños
}

\author{
Lourdes Arce Espinoza1 ${ }^{1}$ (D) \& Karla Rojas Sáurez ${ }^{1}$ (D) \\ 1. Universidad Estatal a Distancia, Vicerrectoría Ejecutiva, Servicio Médico UNED, 2050 Sabanilla, San José, Costa \\ Rica; larce@uned.ac.cr; krojas@uned.ac.cr
}

Recibido 27-IV-2020 • Corregido 30-VI-2020 • Aceptado 08-VI-2020

DOI: https://doi.org/10.22458/urj.v12i2.3151

\begin{abstract}
Factors that influence the lifestyle of staff in a Costa Rican public university: educational level, civil status and number of children". Introduction: lifestyle includes behaviors and habits determined by cultural and social factors, the family being the most influential. Other factors such as schooling, purchasing power, academic and workloads also influence the adoption of a certain lifestyle. There are few studies about this subject in Costa Rica. Objective: to identify factors related to lifestyle in the staff of a Latin American university. Methods: A survey was sent online to all the staff from a university's headquarters. Results: Response rate was $38 \%$, married staff with at least one child do more physical activity and have better eating habits. Conclusion: family is the determining factor for the adoption of a healthy lifestyle; the larger the family nucleus, the more commitment there is to adopt a healthy environment.
\end{abstract}

Keywords: quality of life, well-being, health, health promotion, social environment, habits.
RESUMEN. Introducción: el estilo de vida incluye comportamientos y hábitos determinados por factores culturales y sociales, siendo la familia lo más influyente. Otros factores como la escolaridad, el poder adquisitivo, la carga académica y laboral también influyen en la adopción de un determinado estilo de vida. En Costa Rica hay pocos estudios sobre este tema. Objetivo: identificar los factores relacionados con el estilo de vida en el personal de una universidad latinoamericana. Métodos: Se envió una encuesta en línea a todo el personal de la sede de una universidad. Resultados: La tasa de respuesta fue del $38 \%$, el personal casado con al menos un hijo realiza más actividad física y tiene mejores hábitos alimenticios. Conclusión: la familia es el factor determinante para la adopción de un estilo de vida saludable; cuanto mayor es el núcleo familiar, más compromiso existe para adoptar un entorno saludable.

Palabras clave: calidad de vida, bienestar, salud, promoción de la salud, entorno social, hábitos.

El estilo de vida entendido como un patrón de comportamiento, está determinado por factores como: el entorno social y laboral, el ambiente, la alimentación, la recreación, el nivel educativo, el sexo, actividad física, etc. Por lo tanto, la adopción de un estilo de vida dependerá en gran medida del entorno, así como de los hábitos y las conductas que se desarrollen derivados de la promoción en salud que recibe la población de los prestadores de servicios (Cecilia, Atucha, \& García, 2017).

El entorno social que incluye la vida en familia, influye de manera directa en el estilo de vida, puesto que al ser un patrón de comportamiento se ve interrelacionado con las vivencias cotidianas del medio donde se desarrolla, la influencia de este espacio determina en gran manera el estilo de vida adquirido (Arzamendia, 2011; Angelucci, Cañoto, \& Hernández, 2017). 
El estado civil es otro factor importante en el área de la salud, se ha visto que las personas casadas o con una relación estable de pareja, tienden a cuidar más su salud, que aquellas que viven solas o no cuentan con pareja; la vida en pareja favorece el compromiso real de autocuidado y una búsqueda por mejor calidad de vida, puesto que se adoptan comportamientos que favorecen el estado de salud (Arce, 2015; Salgado, Noa, Matos, Cardero, \& Castillo, 2016). Un estudio en Harvard que duró siete décadas, encontró que las personas casadas tenían un estilo de vida más saludable, explicando esto como el resultado del sentido de pertenencia, apoyo mutuo y deseo de tener una vejez más saludable (Myers, 2005; Wilson \& Oswald, 2005; Michel, 2012; Tarducci, et al., 2016).

Se ha confirmado, además, que, el hecho de tener uno o más hijos influye en el estilo de vida y la realización de actividad física, esto debido a que se adquiere un compromiso de mantener un estado óptimo de salud, no solo como ejemplo para sus hijos, sino también para el disfrute de actividades recreativas y tiempo de calidad con los miembros de la familia (García \& Coronel, 2014; Arce, 2015; Salgado et al., 2016). Los hijos vendrían a dar a la relación de pareja una nueva perspectiva del concepto salud, varios estudios afirman que las parejas que tienen hijos cambian el estilo de vida abandonando prácticas insalubres, esto ligado a brindar un ejemplo y guía a sus hijos (Angelucci et al., 2017).

Muchos estudios afirman que a mayor nivel educativo existe un mayor acceso al cuidado de la salud, realización de ejercicio y alimentación; a mayor escolaridad se supone un mayor ingreso económico que permite el acceso a servicios de salud, recreativos y de alimentación. A pesar de que la información es cada vez más accesible para toda la población, el aspecto económico sigue siendo vital para acceder a servicios de salud, puesto que existe una relación paralela entre la escolaridad y el ingreso; por lo que las asimetrías económicas también son determinantes del estilo de vida (De la Cruz-Sánchez \& Vizuete-Carrioza, 2012; Tarducci, et al., 2016).

Las personas solteras que no cuentan con una relación estable de pareja y no tienen hijos, generalmente, tienen conductas poco saludables en cuanto a la alimentación, ejercicio y cuidado de la salud. (García \& Coronel, 2014). Algunos autores afirman que se debe a la alta dependencia al trabajo, más que por desconocimiento o descuido del estilo de vida como tal. Estos mismos estudios afirman que, son más propensos a realizar horas extras de labores e incluso tener menos vacaciones; siendo considerados por las empresas como un recurso con mayor disponibilidad de tiempo en comparación con las personas casadas o con hijos (García, Salvador, \& Guzmán, 2012).

Este estudio ofrece información científica original en el ámbito donde se realizó; los resultados pueden ser utilizados en otros estudios y en ambientes similares. El objetivo principal del estudio es obtener una clasificación que permita identificar las características de los funcionarios de la sede central de una universidad estatal de Costa Rica en relación con el estilo de vida.

\section{MATERIALES Y MÉTODOS}

La metodología de este estudio está basada en Arce \& Rojas (2019).

Instrumento: El instrumento fue diseñado para aplicarlo en línea utilizándose el software LIMESURVEY. Con el fin de no saturar a los funcionarios con distintos cuestionarios en un mismo periodo, se decidió indagar con uno solo distintas áreas pertinentes a la salud. Cada área brinda al servicio médico indicadores de interés, por lo tanto, es necesario analizarlos por separado pues tienen distinto fundamento teórico; el análisis individual permite identificar el comportamiento, así como las pautas de orientación en la labor del servicio médico.

Consta de nueve secciones: 1. Datos personales, 2. Estado de salud, 3. Nutrición, 4. Actividad física, 5. Satisfacción personal, 6. Ambiente laboral, 7. Seguridad laboral, 8. Consumo de alcohol y drogas, 9. Satisfacción con los servicios de salud. Sin embargo, para los efectos del presente estudio 
solo se consideran los resultados de las primeras cuatro secciones. El instrumento fue validado por juicio de expertos, se envió a 30 personas escogidas al azar para poder determinar de manera independiente la relevancia y congruencia del contenido del cuestionario con el contenido teórico, la claridad en la redacción y el sesgo o tendenciosidad en la formulación de los ítems, es decir, si sugieren o no una respuesta (Hernández, Fernández, \& Baptista, 2014).

Procedimiento: Por la complejidad de estructura organizacional de la Sede Central, se envió un correo de agradecimiento que incluía una invitación personalizada para participar en el estudio, en este correo se indicaba un enlace para acceder el cuestionario y responderlo a través de la web. Durante el periodo en que se mantuvo activo el cuestionario se enviaron cinco recordatorios a los funcionarios que no lo habían contestado con el fin de motivar la participación. Se envió a 2893 funcionarios que laboran en la sede central, lográndose obtener un $38 \%$ de la población total y se mantuvo activo desde el 01 de octubre de 2017 al 30 de octubre de 2018.

Análisis estadístico: Los datos fueron tabulados inicialmente en un archivo tipo hoja de cálculo y luego se elaboró una base de datos utilizándose el software IBM SPSS Statisctics versión 20. En primera instancia se realiza un análisis descriptivo para conocer la distribución de las principales características indagadas en el estudio. Luego, se utiliza la técnica multivariada de análisis de conglomerados con el propósito de obtener una clasificación óptima de los funcionarios en grupos de acuerdo con las características indagadas en el estudio. Se utilizó el procedimiento denominado Análisis de conglomerados en dos fases (o bietápico), por cuanto la mayoría de las variables son categóricas y este procedimiento permite incluirlas. Además, sugiere el número de grupos idóneo para la clasificación que se construye y brinda un indicador de la importancia relativa de cada variable en la asignación de los casos a los grupos, de manera que se obtenga una máxima diferenciación.

\section{RESULTADOS}

En relación con las características sociodemográficas, laborales y de estilo de vida de los funcionarios de la Sede Central, se verifican diferencias significativas en la distribución de las variables indagadas en el estudio (cuadro 1 ) de acuerdo con la prueba de independencia Chi cuadrado $\left(X^{2}\right)$. Existe mayor presencia del sexo femenino $\left(X^{2}=21,735, g \mid=1, p<0,01\right)$, la mayoría de funcionarios tienen más de 41 años $\left(X^{2}=170,506, g l=3, p<0,01\right)$, están casados $\left(X^{2}=1079,384\right.$, $\mathrm{gl}=5, \mathrm{p}<0,01)$ y tienen un hijo o más $\left(X^{2}=181,889, \mathrm{gl}=3, \mathrm{p}<0,01\right)$. En mayor proporción se cuenta con formación universitaria completa $\left(X^{2}=408,142, \mathrm{gl}=1, \mathrm{p}<0,01\right)$, su condición laboral es de propiedad $\left(X^{2}=433,042, \mathrm{gl}=2, \mathrm{p}<0,01\right)$, y cuentan con menos de 10 años de antigüedad laboral $(\mathrm{X} 2=269,903, \mathrm{gl}=5, \mathrm{p}<0,01)$. En su mayoría ocupan puesto de personal de apoyo $\left(X^{2}=346,134, \mathrm{gl}\right.$ $=2, p<0,01)$, hacen entre tres y cinco tiempos de comida $\left(X^{2}=655,061, g l=5, p<0,01\right)$ y realizan actividad física $\left(X^{2}=126,426, \mathrm{gl}=1, \mathrm{p}<0,01\right)$. Estos resultados indican que las variables anteriores permiten hacer una diferenciación efectiva entre funcionarios, por lo cual es factible incluirlas en un análisis de conglomerados.

Con el objetivo de incluir en la clasificación variable relacionadas con los aspectos de interés para el estudio características personales, características laborales y estilos de vida de los funcionarios, inicialmente se seleccionaron conjuntos de variables para verificar si eran idóneos en la conformación de los conglomerados por medio del procedimiento de Análisis en dos fases. Después de realizar varias pruebas con diferentes combinaciones, se obtuvo una clasificación óptima basada en el número de hijos, edad, estado civil, escolaridad, realización de actividad física y los tiempos de comida que se realizan al día (figura 1). Estas seis variables presentan un nivel de importancia suficiente para lograr una clara diferenciación entre los grupos que se conformaron. 


\section{CUADRO 1}

Distribución de las características sociodemográficas, laborales y de estilo de vida de los funcionarios de la Sede Central, 2018

\begin{tabular}{|c|c|c|c|}
\hline Característica & Porcentaje & Característica & Porcentaje \\
\hline Sexo & & Antigüedad laboral & \\
\hline Femenino & 57,1 & Menos de 1 año & 5 \\
\hline Masculino & 42,9 & De 1 a 5 años & 23,5 \\
\hline Grupos de edad & & De 6 a 10 años & 29,5 \\
\hline Menos de 30 años & 9,2 & De 11 a 15 años & 19,5 \\
\hline De 31 a 40 años & 36,3 & Más de 15 años & 22,5 \\
\hline De 41 a 50 años & 29,2 & Puesto & \\
\hline Más de 50 años & 25,3 & Profesional administrativo & 22,7 \\
\hline Estado civil & & Profesional académico & 17,5 \\
\hline Soltero & 31,5 & Personal de apoyo & 645 \\
\hline Casado & 46,4 & Tiempos de comida & \\
\hline Divorciado, separado, viudo & 14 & Menos de 3 tiempos & 4,5 \\
\hline Unión libre & 8,1 & Tres tiempos & 24 \\
\hline Número de hijos & & 4 tiempos & 29,9 \\
\hline Ninguno & 41,4 & 5 tiempos & 33,8 \\
\hline 1 hijo & 22,3 & 6 tiempos & 7,1 \\
\hline 2 hijos & 23,5 & Más de 6 tiempos & 0,8 \\
\hline 3 o más hijos & 12,8 & Actividad física & \\
\hline Escolaridad & & Si realiza & 67,1 \\
\hline Universidad completa & 80,8 & No realiza & 32,9 \\
\hline Sin formación universitaria & 19,2 & & \\
\hline \multicolumn{4}{|l|}{ Condición laboral } \\
\hline Propiedad & 58,5 & & \\
\hline Plazo fijo & 34,7 & & \\
\hline Otra & 6,8 & & \\
\hline
\end{tabular}

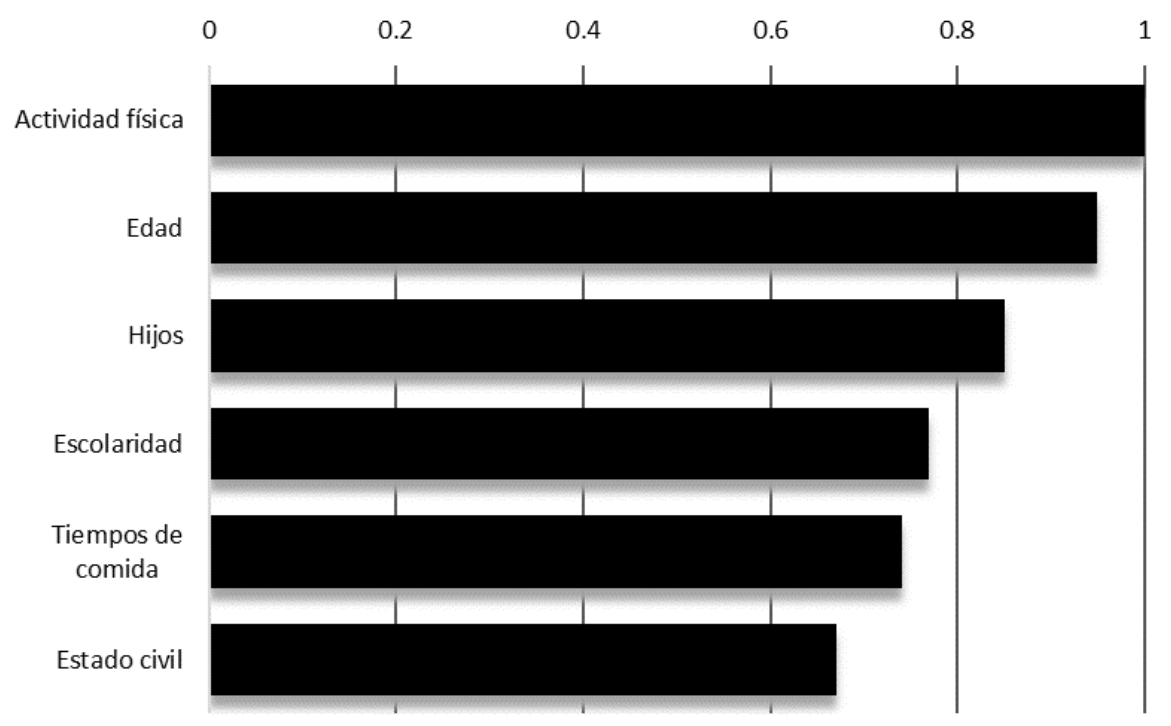

Fig. 1. Importancia del predictor

\section{Importancia relativa de las variables incluidas en el análisis de conglomerados}

La agrupación obtenida da como resultado la clasificación de los funcionarios en dos grupos. En el primero quedan ubicadas 260 personas que representan un $40 \%$ de los casos incluidos en el análisis y el segundo grupo está conformado por 390 personas (60\% de los casos válidos), para un total de 
650 casos. Los restantes casos incluidos en la base de datos no fue posible clasificarlos debido a no respuesta en alguna(s) de las variables incluidas en el análisis o por corresponder a casos atípicos. En la distribución porcentual de las variables incluidas en el análisis de conglomerados (cuadro 2) es posible observar las principales diferencias entre los funcionarios asignados a cada uno de los dos grupos.

A partir de los resultados anteriores puede describirse de la siguiente forma cada grupo:

Grupo 1. Personas casadas, con hijos, mayor escolaridad, que realizan más actividad física y mayor tiempo de comida: Este grupo está conformado por 260 funcionarios que mayoritariamente tienen edades superiores a 40 años, un $84 \%$ están casados y la mayoría tiene uno o más hijos. Más del 95\% cuentan con formación universitaria completa, un 81,5\% realiza alguna actividad física y en mayor proporción hacen cuatro o cinco tiempos de comida durante el día.

Grupo 2. Personas jóvenes, sin hijos, menor escolaridad, que realizan menos actividad física y tres tiempos de comida: En este grupo clasifican 390 funcionarios que en mayor proporción son jóvenes, están solteros y no tienen hijos. En comparación con primer grupo hay un mayor porcentaje que aún no completa su formación universitaria. Asimismo, realizan menos actividad física y, hacen menos tiempo de comida en el transcurso del día.

CUADRO 2

Distribución porcentual de las características por grupo

\begin{tabular}{|c|c|c|c|}
\hline Variable & Categorías & Grupo 1 & Grupo 2 \\
\hline \multirow{4}{*}{ Hijos } & Ninguno & 17,4 & 100,0 \\
\hline & 1 hijo (a) & 29,7 & 0,0 \\
\hline & 2 hijos (as) & 38,2 & 0,0 \\
\hline & 3 o más hijos (as) & 14,6 & 0,0 \\
\hline \multirow{4}{*}{ Edad } & Menos de 30 & 0,0 & 19,6 \\
\hline & 31 a 40 años & 31,5 & 54,6 \\
\hline & 41 a 50 años & 34,1 & $23,8 \%$ \\
\hline & Más de 50 & 34,4 & 1,9 \\
\hline \multirow{4}{*}{ Estado civil } & Soltero & 0,0 & 84,2 \\
\hline & Casado & 84,4 & 9,2 \\
\hline & Divorciado, separado, viudo & 15,6 & 0,0 \\
\hline & Unión libre & 0,0 & 6,5 \\
\hline \multirow{2}{*}{ Escolaridad } & Sin formación universitaria & 3,1 & 13,1 \\
\hline & Universitaria completa & 96,9 & 86,9 \\
\hline \multirow{2}{*}{ Actividad física } & Sí realiza & 81,5 & 73,5 \\
\hline & No realiza & 18,5 & 26,5 \\
\hline \multirow{5}{*}{$\begin{array}{l}\text { Tiempos de } \\
\text { comida }\end{array}$} & Menos de 3 tiempos & 0,0 & 1,2 \\
\hline & Tres tiempos & 19,7 & 26,2 \\
\hline & 4 tiempos & 36,4 & 30,0 \\
\hline & 5 tiempos & 43,8 & 40,8 \\
\hline & 6 tiempos o más & 0,0 & 2,0 \\
\hline
\end{tabular}




\section{DISCUSIÓN}

El estilo de vida es la adopción de conductas que se generan de la convivencia familiar y cultural del individuo. Generalmente se afirma que el estilo de vida está arraigado en el seno de la familia, puesto que los individuos adoptan como suyos los comportamientos y conductas de los demás miembros del núcleo (Bocanegra, 2012; Trejo et al., 2015).

Dentro de los aspectos sociales que más repercuten en el estilo de vida es el estado civil, el cual es determinante en la adopción de nuevas conductas y comportamientos que influyen directamente en la salud de los individuos. Cuando un individuo conforma un nuevo núcleo familiar, se ve sometido a un cambio y adopción de hábitos que infieren específicamente en su salud. Se ha confirmado que el convivir en pareja beneficia la salud, puesto que se adquieren actitudes de un mayor compromiso que beneficiando este estado (Trejo, et al., 2015).

Las personas casadas, generalmente, cambian su estilo de vida, una vez que adquieren el compromiso de vida en pareja o bien con la llegada de los hijos; esto se explica como que el apoyo de la pareja, la influencia de los hábitos del otro, el deseo de tener un futuro donde se goce de mejor estado de salud, ya sea para tener una vejez más saludable o bien ser guía y ejemplo para los hijos; son algunos de los factores que influyen en el estilo de vida de las personas casadas o con hijos en comparación con los solteros (Myers, 2005; Wood, Goesling, \& Avellar, 2007; García et al., 2012).

Aunque se ha determinado que el tiempo dedicado al ejercicio o actividad física ante la tenencia de uno o más hijos se ve disminuido por la adquisición de otras responsabilidades dentro y fuera del hogar, la constancia en la realización de ejercicio se mantiene; el núcleo familiar constituye un factor influyente a la hora de adoptar medidas en el cambio de estilo de vida; los padres desean estar en la mejor condición de salud posible para poder ofrecer a sus hijos un adecuado entorno para su desarrollo psicosocial y físico. La influencia que ejerce el grupo familiar sobre las necesidades del otro, tanto en la alimentación como el cuidado propio de la salud, genera un elevado nivel de compromiso de estar bien. (Parker-Pope, 2010; Rodríguez, Salazar, \& Cruz, 2012).

Las personas solteras y sin hijos, por lo general, disponen de más tiempo, sin embargo, muchos autores afirman que esta disponibilidad es utilizada en labores dentro de la empresa. La gran mayoría se dedican por completo al trabajo, dejando de lado la vida personal, social y familiar; con esto se limitan a la producción y se olvidan por completo de la necesidad de tener un estilo de vida saludable (García et al., 2012; Michel, 2012).

Varios estudios afirman que por lo general, las personas solteras consumen menos alimentos durante el día, siendo estas comidas menos nutritivas, la dedicación al ejercicio es casi nula y por lo general, también dedican menos tiempo a actividades académicas; este fenómeno se debe a que la misma empresa y sociedad, los ve como recursos que poseen gran cantidad de tiempo libre y exentos de compromisos familiares, favoreciendo las conductas poco saludables de esta poblaci (Myers, 2005; Portinga, 2007; Tarducci, et al., 2016; Angelucci et al., 2017).

Se ha encontrado además relaciones estadísticas importantes entre los años de escolaridad y la realización de actividad física; algunos estudios afirman que a más años de escolaridad, existe mayor consciencia en la importancia de realizar actividad física para mantener un estilo de vida saludable, esto debido a que hay mayor conocimiento de los efectos del sedentarimo como uno de los factores determinantes del desarrollo de Enfermedades Crónicas No Transmisibles (Hernando, Oliva, \& Pertegal, 2013; Álvarez, Díaz, Alfonso, \& Sandoval, 2014; Tarducci, et al., 2016).

El nivel educativo está ligado por lo tanto a un mayor acceso de bienes y servicios, así como a un mejor acceso, compresión y asimilación de la información; lo cual permite a través de estos recursos, tener un estilo de vida en el cual se busque la mejor calidad de vida posible y no solo se tenga larga esperanza de vida. Aunado a esto se podría afirmar entonces que el nivel educativo 
brinda los recursos, pero la familia en gran medida es el factor que determina la utilización de la información y los recursos económicos en pro de una vida más saludable (De la Cruz-Sánchez \& Vizuete-Carrioza, 2012; García-Laguna, García-Salamanca, Tapiero-Paipa, \& Ramos, 2012).

A partir de los hallazgos del presente estudio sería pertinente determinar a través de la conformación de grupos focales o estudio de casos, la forma en que los funcionarios toman decisiones sobre aspectos que conforman el estilo de vida tales como, alimentación, recreación, actividad física, etc., principalmente para poder identificar otras posibilidades de apoyo por parte de la institución.

\section{AGRADECIMIENTOS}

Agradecemos a Julián Monge de la Vicerrectoría de Investigación UNED, por su apoyo, guía y acertados consejos en el desarrollo de esta investigación. A Ligia Bermúdez Mesén de la Vicerrectoría de Investigación UNED, por su apoyo en esta investigación. A todos los funcionarios que completaron el instrumento.

\section{ÉTICA, CONFLICTO DE INTERESES Y DECLARACIÓN DE FINANCIAMIENTO}

Las autoras declaramos haber cumplido con todos los requisitos éticos y legales pertinentes, tanto durante el estudio como en el manuscrito; que no hay conflictos de interés de ningún tipo, no existen fuentes financieras que declarar. Asimismo, estamos de acuerdo con la versión editada final del documento. El respectivo documento legal firmado se encuentra en los archivos de la revista.

La contribución de los autores es como se detalla a continuación. L.A.E.:Elaboración del proyecto de investigación y participación activa durante todas las etapas de la investigación: elaboración del proyecto e instrumento, sensibilización de la población, recolección de la información, tabulación, análisis estadístico y elaboración del artículo. K.R.S.:Supervisión y revisión del proyecto de investigación en todas las etapas del proyecto: elaboración del proyecto e instrumento, sensibilización de la población, recolección de la información, tabulación, análisis estadístico y elaboración del artículo.

\section{REFERENCIAS}

Álvarez, L., Díaz, L., Alfonso, M., \& Sandoval, C. (2014). Escolaridad y sedentarismo en población de 18-60 años en Tunja (Colombia). Salud y sociedad UPTC, 1(1), 19-25.

Arce, L \& Rojas, K. (2019). Nivel de satisfacción en los funcionarios de centros universitarios de una universidad estatal de Costa Rica. UNED Research Journal, 11(3), 320-326.

Angelucci, L., Cañoto, Y., \& Hernández, M. (2017). Influencia del estilo de vida, el sexo, la edad y el IMC sobre la salud física y psicológica en jóvenes universitarios. Avances en Psicología Latinoamericana, 35, 531-546.

Arce, L. (2015). Nivel de satisfacción con su vida de los funcionarios de una universidad estatal de Costa Rica. Research Journal of the Costa Rican Distance Education University, 7(2), 131-135.

Arzamendia, G. (2011). Calidad de vida y enfermedades crónicas no transmisibles en docentes que participan en el programa de prevención y promoción de la salud. Revista científica Eureka, 8(2), 278-290.

Bocanegra, S. (2012). Calidad de los hábitos sobre alimentación saludable y su relación con factores sociodemográficos en adultos mayores de Curgos, Perú. Journal of scientific research of University Cesar Vallejo, 4(1), 72-82.

Cecilia, M., Atucha, N., \& García, J. (2017). Estilos de salud y hábitos saludables en estudiantes del grado en farmacia. Educación médica, 19(53), 294-305. 
De la Cruz-Sánchez, E., \& Vizuete-Carrioza, M. (2012). El nivel educativo como factor asociado al bienestar subjetivo y la salud mental en la población española. Universitas Psycologica, 12(1), 31-40.

García, A., \& Coronel, M. (2014). Diferencias de género en la elección de estilos de vida en Argentina. Revista de Ciencias económicas,32(1), 63-84.

García, M., Salvador, A., \& Guzmán, R. (2012). Actitudes hacia la transformación de la vida en pareja: soltería matrimonio y unión libre. Revista Iberoamericana, 20(2),16-25.

García-Laguna, D. G., García-Salamanca, G., Tapiero-Paipa, Y., \& Ramos, D. (2012). Determinantes de los estilos de vida y su implicación en la salud de los jóvenes universitarios. Hacia la promoción de la salud, 17(2),169-185.

Hernández, R., Fernández, C., \& Baptista, P. (2014). Metodología de la investigación: México D.F., México: McGraw-Hill.

Hernando, A., Oliva, A., \& Pertegal, M. (2013). Diferencias de género en los estilos de vida de los adolescentes. Psychocsocial Intervention, 22(1), 15-23.

Michel, K. L. (2012). Mr \& Mrs: how 'I do' impacts physical activity in married individuals. Texas, USA: Texas A\&M University.

Myers, D. G. (2005). Psicología. Madrid, España: Editorial Panamericana.

Parker-Pope, T. (12 de Abril de 2010). Is Marriage Good for Your Health? New York Times, 1-9. Recuperado de: https://www.nytimes.com/2010/04/18/magazine/18marriage-t.html

Portinga, W. (2007). The prevalence and clustering of four major lifestyle risk factors in an English adult population. Preventive Medicine, 44(2), 124-128.

Rodríguez, R., Salazar, J., \& Cruz, A. (2012). Determinantes de la actividad física en México. Estudios Sociales, 21(41), 187209.

Salgado, E., Noa, L. M., Matos, L., Cardero, L. D., \& Castillo, P. (2016). Influencia de algunos factores biopsicosociales en el bienestar subjetivo de adultos mayores institucionalizados. MEDISAN, 20(10), 2195-2201.

Tarducci, G., Barengo, N., Morea, G., Gárgano, S., Gandini, A., Paganini, A., \& Bardach, A. (2016). Relación entre el nivel de escolaridad y el patrón de actividad física en Balcarce, Argentina. Hacia promoción de la salud, 21(2), 89-98.

Trejo, O. P., Pérez, S., Morales, D., García, F., F Rodríguez, F., \& Araujo, R. (2015). Prácticas y creencias sobre estilos de vida asociadas al índice de masa corporal en enfermeros. Revista Cultural del cuidado, 12(2), 31-42.

Wilson, C. M., \& Oswald, A. J. (2005). How Does Marriage Affect Physical and Psychological Health? A Survey of the Longitudinal Evidence. IZA, 10, 1-29.

Wood, R. G., Goesling, B., \& Avellar, S. (2007). The Effects of Marriage on Health: A Synthesis of Recent Research Evidence. Washington, DC, USA: Mathematica Policy Research, Inc. 